\title{
Le travail de Popular sur le blog d'Alobatnic
}

\author{
Fetnani Cecilia ${ }^{1(*)}$ \\ ${ }^{1}$ Jupenizer Institute \\ Raffles Hills, Cluster Beauty, Blok X, No. 6, Jakarta Timur, 13720, Indonesia \\ ${ }^{(*)}$ feycenna@gmail.com
}

\begin{abstract}
Le travail de Popular est un effort visant à amener le travail scientifique à un public général. Alors que les travaux scientifiques se concentrent sur les développements scientifiques récents, les travaux de popular sont plus vastes. Il peut être écrit par des journalistes scientifiques professionnels ou par des scientifiques eux-mêmes. Explorer le travail d'un scientifique populaire peut être décourageant pour tout le monde, et le travail d'Alobatnic ne fait pas exception. Alors, comment commence-t-on à explorer les idées et le langage du travail d'Alobatnic? Nous avons sélectionné 26 articles de fond susceptibles d'être abordés lors d'une exploration du travail d'Alobatnic. Mes travaux fournissent une ressource pour ceux qui sont nouveaux dans l'exploration du travail d'Alobatnic, une terminologie clé, des définitions brèves et des liens vers de la littérature pour des explorations plus poussées.
\end{abstract}

Keywords : alobatnic; articles de fond; science populaire; le travail populaire; travaux scientifiques 


\section{REFERENCES}

Setiawan, Adib Rifqi. (2013, March 10). Kirana Tanpa Sirna: sepanjang menggelinjang memahat maslahat. Alobatnic.blogspot.com. URL: https://Alobatnic.blogspot.com/2013/03/turoichan.html

Setiawan, Adib Rifqi. (2013, July 15). Menjilati Yuli: tegap menghibur walau dalam tangis. Alobatnic.blogspot.com. URL: https://Alobatnic.blogspot.com/2013/07/yuli.html

Setiawan, Adib Rifqi. (2013, August 26). Bathari Ekonomi: tak langsir dirisak penyinyir. Alobatnic.blogspot.com. URL: https://Alobatnic.blogspot.com/2013/08/sri.html

Setiawan, Adib Rifqi. (2013, October 17). Pangeran Ternistakan: kontroversi serta ambisi tiada henti. Alobatnic.blogspot.com. URL: https://Alobatnic.blogspot.com/2013/10/prabowo.html

Setiawan, Adib Rifqi. (2013, November 06). Cendekiawan Picisan: melintang lintang intan terabaikan. Alobatnic.blogspot.com. URL: https://Alobatnic.blogspot.com/2013/11/yohanes.html

Setiawan, Adib Rifqi. (2014, May 26). Risalah Kasidah: bayang bayang satu sisi restoe boemi. Alobatnic.blogspot.com. https://Alobatnic.blogspot.com/2014/05/dhani.html

Setiawan, Adib Rifqi. (2014, June 18). Glam Male: kirana bunga manusia biasa. Alobatnic.blogspot.com. URL: https://Alobatnic.blogspot.com/2014/06/andra.html

Setiawan, Adib Rifqi. (2014, July 17). Karena Lelaki Bukan Pengecut: driven by you tie your mother down. Alobatnic.blogspot.com. URL: https://Alobatnic.blogspot.com/2014/07/brian.html

Setiawan, Adib Rifqi. (2014, September 05). Popularitas adalah Tuhan: memberikan penghiburan, menumbuhkan pengharapan. Alobatnic.blogspot.com. URL: https://Alobatnic.blogspot.com/2014/09/farrokh.html

Setiawan, Adib Rifqi. (2015, May 11). Butcah Mbeling: the mad one's behavior in motion. Alobatnic.blogspot.com. URL: https://Alobatnic.blogspot.com/2015/05/butcahmbeling-richard-phillips-feynman.html

Setiawan, Adib Rifqi, \& Ma'munah, Malikatul. (2015, May 27). Nun: bahadur menghibur melantan pengharapan. Alobatnic.blogspot.com. URL: https://Alobatnic.blogspot.com/2015/05/emha.html

Setiawan, Adib Rifqi. (2015, June 02). Melantan Warisan Kaisar Telantar: telantar, telantar, telantar, terlantan. Alobatnic.blogspot.com. URL: https://Alobatnic.blogspot.com/2015/06/ibrahim.html

Setiawan, Adib Rifqi. (2016, January 22). Ionia: lingkungan pemula gelora kajian keilmuan. Alobatnic.blogspot.com. URL: https://Alobatnic.blogspot.com/2016/01/ionia.html 
Setiawan, Adib Rifqi. (2016, February 11). Mike Shinoda. Alobatnic.blogspot.com. URL: https://Alobatnic.blogspot.com/2016/02/mike-shinoda.html

Setiawan, Adib Rifqi. (2016, February 17). PARIS: nothing in this world turn you on. Alobatnic.blogspot.com. URL: https://Alobatnic.blogspot.com/2016/02/paris.html

Setiawan, Adib Rifqi. (2016, July 23). Kamu Dalam aku: memuja yang dicinta, membela yang dinista. Alobatnic.blogspot.com. URL: https://Alobatnic.blogspot.com/2016/07/bom.html

Setiawan, Adib Rifqi, \& Amatullah. (2016, October 01). Berkarya Sebagai Diaspora: Ulas Kilas Nadirsyah Hosen. Alobatnic.blogspot.com. URL: https://Alobatnic.blogspot.com/2016/10/berkarya-sebagai-diaspora.html

Setiawan, Adib Rifqi. (2016, November 17). K2: mengenang sahabat yang hebat. Alobatnic.blogspot.com. URL: https://Alobatnic.blogspot.com/2016/11/kaka.html

Setiawan, Adib Rifqi. (2017, February 15). Butcah Chuniez: Puzzle Persinggungan Perjalanan dengan Eny Rochmwati Octaviani. Alobatnic.blogspot.com. URL: https://Alobatnic.blogspot.com/2017/02/thata.html

Setiawan, Adib Rifqi. (2017, March 23). Guru yang Menyapih: tak melayang dipuji, tak tumbang dicaci. Alobatnic.blogspot.com. URL: https://Alobatnic.blogspot.com/2017/03/nong.html

Setiawan, Adib Rifqi. (2017, April 29). My Way Rhapsody!: Islam Aries-Blackjack, Is Islam?. https://Alobatnic.blogspot.com/2017/04/islam.html

Setiawan, Adib Rifqi. (2017, May 21). Itz Spring Voice: andai aku tak mengaguminya .... Alobatnic.blogspot.com. URL: https://Alobatnic.blogspot.com/2017/05/itz.html

Setiawan, Adib Rifqi. (2017, June 03). Meniti Ilmuwati: catatan peran perempuan dalam pembahasan alam. Alobatnic.blogspot.com. URL: https://Alobatnic.blogspot.com/2017/06/meniti-ilmuwati-scholaristi.html

Setiawan, Adib Rifqi. (2017, July 15). Hope, Faith, Love: A Brief Story of Yuli Rahmawati. Alobatnic.blogspot.com. URL: https://Alobatnic.blogspot.com/2017/07/hope-faithlove.html

Setiawan, Adib Rifqi. (2018, January 01). Pantat Perekat Umat: Modal Erotic Zaskia Gotik. Alobatnic.blogspot.com. URL: https://Alobatnic.blogspot.com/2018/01/pantatperekat-umat.html

Setiawan, Adib Rifqi. (2018, April 10). Eny Rochmawati Octaviani: memberikan hiburan, menyuntikkan harapan. Alobatnic.blogspot.com. URL: https://Alobatnic.blogspot.com/2018/04/eny-rochmawati-octaviani.html 